\section{Karen Ritchie Jean-Marie Robine}

\section{ADRESSE}

K. Ritchie: changée de recherche à l'Inserm. J.M. Robine: chargé de recherche à l'Inserm. CRLC, Val d'Aurelle, Parc Euromédecine,

\title{
La démence sénile en France: une estimation de la charge actuelle et projection des tendances futures
}

L'estimation, à partir des données du Recensement général de la population de 1990 , du nombre de personnes souffrant actuellement de démence sénile en France, suggère que le nombre de cas en 1990 est moins élevé que ce qui avait été estimé précédemment. Une augmentation significative est toutefois attendue dans les années à venir si aucun traitement efficace de la démence n'est découvert ou si l'âge moyen de survenue n'est pas reculé. L'importance de ces prévisions, en termes de santé publique et de programmation sanitaire, va bien au-delà de la prévision de l'aide requise pour les personnes âgées démentes ; les implications d'une telle épidémie concernent également les familles qui prennent souvent en charge les personnes démentes.

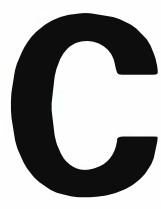
omme la chute de la mortalité concerne principalement les âges élevés, les gestionnaires des services de santé ont fortement exprimé leur crainte de voir le vieillissement consécutif de la population apporter avec lui toute une épidémie de maladies chroniques invalidantes. En particulier, ces craintes ont été alimentées par l'augmentation projetée de la prévalence de la démence sénile. Ces troubles qui impliquent une détérioration progressive de toutes les fonctions intellectuelles, entraînent une perte de l'autonomie dans la réalisation des performances du fonctionnement quotidien, chez des per- sonnes dont la durée moyenne de survie est estimée à environ huit ans. Comme la démence sénile implique une perte progressive du jugement, l'individu atteint devient incapable d'apprécier l'importance de ses incapacités. La démence sénile est donc un vrai problème de santé publique et l'observation d'un changement dans la prévalence peut être d'une importance considérable pour la programmation des services de santé.

Les quelques tentatives qui ont été faites à ce jour pour prévoir la charge que représenteront pour la société les maladies démentielles à la fin du siècle ont toutes abouti à des résultats très pessimistes. Inei- 
chen [1] annonce une "marée montante " des démences au RoyaumeUni et estime que l'augmentation de la prévalence sera comprise entre $5 \%$ et $17 \%$ d'ici 2001 . Henderson [2] signale "l'arrivée d'une épidémie de démences " en Australie, estimant à $91 \%$ l'augmentation du nombre de cas d'ici 2001. En France, Mas et al. [3] ont prédit un accroissement d'environ $33 \%$ des cas d'ici l'an 2010, uniquement pour la maladie d'Alzheimer. Jorm [4] a noté que le pourcentage d'augmentation du nombre de cas, d'ici la fin du siècle, variera fortement en fonction de l'évolution des tendances de mortalité aux différents âges. Il a, en outre, estimé les taux de démences pour plusieurs pays, en utilisant des projections de populations issues des Nations Unies pour l'année 1980, et des prévalences estimées à partir d'un large éventail d'études. Malheureusement, ces études ont des critères diagnostiques très variés et comportent un nombre insuffisant de sujets appartenant aux groupes d'âges les plus élevés. De telles projections ont toutes les chances d'être inexactes dans la mesure où elles ne prennent pas en compte les plus importantes sources d'erreurs.

L'estimation du nombre de cas de démences séniles varie selon la nature de l'étude réalisée. Ces variations dépendent, en premier lieu, du niveau de démence considéré. Les études épidémiologiques de la démence sénile tendent, en fait, à se limiter aux formes de démence modérée ou sévère. La raison en est double. D'abord, les démences légères n'ont pas de retentissement significatif sur les activités quotidiennes. Ensuite, l'estimation de la prévalence de la démence sénile légère est peu précise, avec des taux variant largement entre les différentes études conduites au sein d'un même pays [5]. En revanche, il existe actuellement une grande cohérence dans l'estimation de la prévalence de la démence modérée ou sévère [6].

L'estimation de la prévalence de la démence sénile varie aussi avec la définition retenue. Les études de prévalence conduites depuis les années 80 tendent à n'utiliser que des algorithmes diagnostiques inter- nationalement reconnus, tel celui du manuel diagnostique de l'American Psychiatric Association (DSM III) [7] ou de la Classification internationale des maladies [8]. Les taux obtenus sont alors largement convergents à travers les pays d'Europe de l'Ouest et d'Amérique du Nord. Les études réalisées précédemment utilisaient des procédures de dépistage des cas fortement variables qui aboutissaient à des taux de prévalence peu cohérents au sein d'un même pays [6]. Par exemple, Essen-Möller et al. [9] rapportent pour la Suède un taux global de prévalence de la "détérioration sénile " de 15,8\% au-delà de 65 ans alors qu'Akesson et al. [10] trouvent, pour le même groupe d'âge, un taux de prévalence de la démence de $1,3 \%$. Depuis les années 80 , la tendance générale a été d'adopter des algorithmes diagnostiques des cas internationalement acceptés, avec comme résultat la constatation générale d'une prévalence de la démence sénile (modérée et sévère) d'environ $5 \%$ au-delà de 65 ans aussi bien en Europe qu'aux États-Unis ou au Japon.

Une troisième source de variations est liée aux techniques d'échantillonnage. Étant donné le taux élevé de placements en institution associés aux démences séniles, toute estimation basée sur la population des ménages ordinaires sous-estime gravement le nombre de personnes atteintes de démence sénile dans la population générale. En outre, la tendance des études épidémiologiques à ne pas ajuster les taux de sondage en fonction de la diminution des effectifs dans les strates d'âges les plus élevées aboutit à accroître l'imprécision des estimations aux âges où, à la fois, la prévalence de la démence et les risques de mortalité sont au plus haut. Ainsi, de petites erreurs peuvent avoir un effet important sur l'estimation du nombre total de personnes souffrant de démence.

Une autre source d'erreur vient s'ajouter aux estimations quand des tentatives sont faites pour prédire l'évolution future du nombre de cas de démence. En effet, l'imprécision des projections de population constitue une source supplémentaire d'erreur considérable. Ainsi, jusqu'à présent, les démographes ont quasi systématiquement sous-estimé, dans leurs scénarios de projection, la chute à venir de la mortalité, cela d'autant plus que l'âge considéré est élevé.

Ainsi, en 1975, la seule hypothèse de mortalité étudiée par l'INSEE pour ses projections de population 1975-2020 était une hypothèse tendancielle : “La mortalité est supposée évoluer dans le prolongement des tendances observées au cours des vingt-cinq dernières années [11]. "En 1985, ayant déjà constaté l'importante sous-estimation du nombre de personnes âgées entraînée par le choix de cette hypothèse, l'INSEE introduisit deux nouvelles hypothèses de mortalité dans ses projections révisées. La première, qualifiée de tendancielle, "se traduit par un gain d'environ deux ans (en termes d'espérance de vie à la naissance) d'ici l'an 2000, puis encore d'environ un an de 2000 à 2020. Après 2020, la mortalité est maintenue constante " [12]. La deuxième hypothèse, qualifiée de basse (bas niveau de mortalité), conduit "à un gain d'espérance de vie d'ici à 2020 environ deux fois plus fort que dans l'hypothèse tendancielle" [12]. En 1990, à nouveau l'INSEE ne retient qu'une hypothèse tendancielle. « La technique adoptée consiste à prolonger, pour chaque âge, le taux moyen annuel de baisse observé dans la période 1970-1990, avec quelques corrections... C'est en fait une actualisation de l'alternative "mortalité basse" des projections de 1985 qui se sont avérées très proches de l'évolution réelle pour la période 1985-1990 [13]. " Ainsi, de 1985 à 1990, c'est l'hypothèse basse des projections de 1985 qui a le mieux correspondu à l'évolution réelle, mais l'INSEE ne retient à partir de 1990 qu'une hypothèse tendancielle comme en 1975.

Il est donc important de fournir une nouvelle estimation du nombre de personnes âgées de plus de 60 ans souffrant d'une démence sénile en France et d'en prévoir l'évolution jusqu'en 2020, dans la mesure où cela est aujourd'hui possible, et compte tenu de toutes les difficultés mentionnées ci-dessus. 


\section{REFERENCES}

1. Ineichen B. Measuring the rising tide how many dementia cases will there be by 2001 ? Br J Psychiatry 1987 ; 150 : 193-200.

2. Henderson AS. The coming epidemic of dementia. Austr N Zeal I Psychiatry 1983; 17: $117-27$

3. Mas JL, Alperovitch A, Derouesne C. Épidémiologie de la démence de type $\mathrm{Al}$ heimer. Rev Neurol 1987; 143: 161-71.

4. Jorm AF. The epidemiology of Alzheimer's disease and related disorders. Melbourne: Chapman and Hall, 1990.

5. Henderson AS, Huppert FA. The problem of mild dementia. Psychol Med 1989; 14: 5-11

6. Ritchie K, Kildea D, Robine JM. The relationship between age and the prevaence of senile dementia : a meta-analysis of recent data. Int J Epidemiol 1992; 21 763-9.

7. American Psychiatric Association. Diagnostic and statistical manual of mental disorders, third adition, American Psychiatric Association, Washington, 1980

8. World Health Organization. Mental disorders: glossary and guide to their classification in accordance with the ninth revision of the international classification of diseases, World Health Organization, Geneva, 1978

9. Essen-Möller E, Larsson $\mathrm{H}$, Uddenberg CE, White (;. Individual traits and morbidity in a Swedish rural population. Acta P.SY Neurol Scand 1956; 100: Suppl.

10. Akesson HO. A population study of senile and arteriosclerotic psychoses. Hum Heredity 1969 ; 19 : 546-66.

11. Dinh QC, Labat JC. Projection de la population totale pour la France, 1975-2020. INSEE, Collections de l'INSE, 1979, Série D, $\mathrm{n}^{\circ} 63$.

12. Dinh QC, Labat JC. Projection de la population totale pour la France, 1985-2040. INSEE, Collections de l'INSEE, 1986, Série D, n 113 .

13. Dinh QC. Projection de la population totale pour la France métropolitaine, Base RP90. Horizon 1990-2020. INSEE, 1992, Document de travail F9213.

14. Jorm AF, Korten AE. A method for calculating projected increases in the number of dementia sufferers - with application to Australia and New Zealand. Austr $N$ Zeal J Psychiatry 1988; 22: 183-9.

15. Gruenberg EM. The failures of success. Milbank Memorial Fund Cuarterly 1977 ; 55 3-24.

16. Kramer M. The rising pandemic of mental disorders and associated chronic diseases and disabilities. Acta Psy Scand $1980 ; 62$ : 282-97.
Figure 1. Prévalence de la démence. Sont comparées les prévalences de l'ensemble des démences (courbe rouge) et de la maladie d'Alzheimer (courbe noire). La prévalence de la démence double tous les six ans. Au-delà de 95 ans la maladie d'Alzheimer rend compte de toutes les démences séniles.

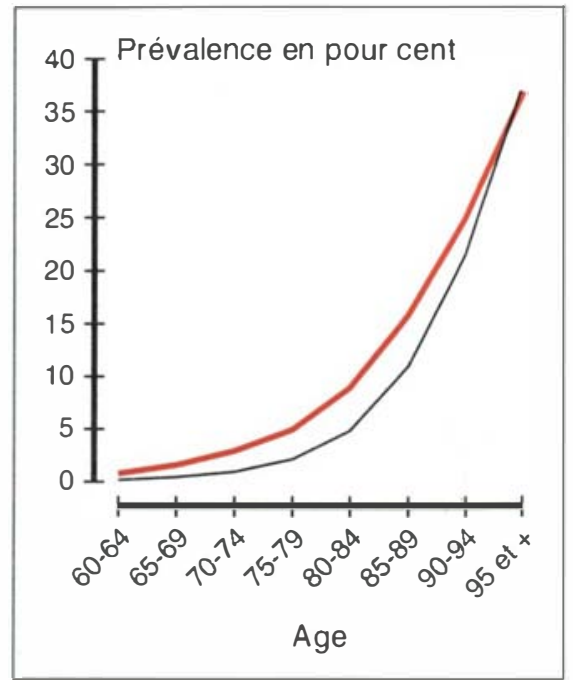

Tableau

PRÉVALENCE DE LA DÉMENCE SÉNILE ET DE LA MALADIE D'ALZHEIMER, EN POURCENTAGE DE LA POPULATION (MODÉLE DE RITCHIE ET AL. [26])

\begin{tabular}{|c|c|c|}
\hline Age & $\begin{array}{c}\text { Démence } \\
\text { sénile }\end{array}$ & $\begin{array}{c}\text { Maladie } \\
\text { d'Alzheimer }\end{array}$ \\
$60-64$ & 0,9 & 0,2 \\
$65-69$ & 1,6 & 0,4 \\
$70-74$ & 2,8 & 0,9 \\
$75-79$ & 4,9 & 2,1 \\
$80-84$ & 8,7 & 4,7 \\
$85-89$ & 15,5 & 10,6 \\
$90-94$ & 24,5 & 21,0 \\
95 et + & 36,7 & 37,4 \\
\hline
\end{tabular}

Tableau II

ESTIMATION DU NOMBRE DE CAS DE DÉMENCE SÉNILE EN FRANCE DE 1990 A 2020 (MODELE RITCHIE ET AL. [26], APPLIQUÉ AUX PROJECTIONS DE POPULATION INSEE (1992) [13]

\begin{tabular}{|c|r|r|r|r|}
\hline Age & 1990 & 2000 & 2010 & \multicolumn{1}{|c|}{$\mathbf{2 0 2 0}$} \\
$60-64$ & 26029 & 24364 & 33411 & 35005 \\
$64-69$ & 43350 & 43911 & 39950 & 59774 \\
$70-74$ & 37065 & 69299 & 66816 & 93943 \\
$75-79$ & 85933 & 103767 & 110189 & 104344 \\
$80-84$ & 105287 & 75765 & 151674 & 154914 \\
$85-89$ & 98047 & 128254 & 171609 & 198886 \\
$90-94$ & 49106 & 80176 & 67141 & 150616 \\
95 et + & 15226 & 30023 & 48688 & 73652 \\
& & & & \\
Total & 460043 & 555558 & 689479 & 871136 \\
$>65$ & 434014 & 531195 & 656067 & 836131 \\
$>85$ & 162379 & 238453 & 287437 & 423155 \\
\hline
\end{tabular}




\section{Estimation de la prévalence de la démence}

L'estimation de la prévalence spécifique de la démence sénile, à un âge donné, au-delà de 60 ans, provient d'une méta-analyse. Il s'agit d'une méthode quantitative, permettant d'agréger des résultats d'études indépendantes. Cette analyse est fondée sur treize études conduites depuis 1980, qui ont utilisé des critères diagnostiques internationalement reconnus, et qui fournissent des données par groupe d'âges [14]. Un modèle linéaire a été développé à partir des logarithmes de la pro- portion des cas. Les données de chaque étude ont alors été ajustées à ce modèle (l'âge ayant été remplacé par la valeur médiane des intervalles quinquennaux de 65 à 89 ans ; à 90 ans et au-delà, l'âge a été remplacé par la valeur de 95 ans). Le modèle suivant a ainsi été obtenu :

$\log (p)=-12,43 \quad(0,62)+0,121$ $(0,007853)$ âge (modèle 1$)$

(les erreurs standards figurant entre parenthèses), sans qu'il y ait de différences significatives (que ce soit pour les pentes ou l'ordonnée à l'origine) entre les différentes études. $\mathrm{R}^{2}$ (pourcentage de la variabilité expliquée par le modèle) est de

Tableau IIII

ESTIMATION DU NOMBRE DE CAS DE MALADIE D'ALZHEIMER EN FRANCE DE 1990 A 2020 (MODĖLE RITCHIE ET AL. [26], APPLIQUÉ AUX PROJECTIONS DE POPULATION INSEE (1992) [13]

\begin{tabular}{|c|r|r|r|r|}
\hline Age & 1990 & 2000 & \multicolumn{1}{|c|}{2010} & \multicolumn{1}{|c|}{2020} \\
$60-64$ & 5784 & 5414 & 7425 & 7779 \\
$64-69$ & 10837 & 10978 & 9988 & 14944 \\
$70-74$ & 11914 & 22275 & 21477 & 30196 \\
$75-79$ & 36828 & 44471 & 47224 & 44719 \\
$80-84$ & 56879 & 40930 & 81939 & 83689 \\
$85-89$ & 68317 & 89364 & 119573 & 138579 \\
$90-94$ & 42091 & 68722 & 57549 & 129100 \\
95 et + & 15517 & 30596 & 49616 & 75057 \\
& & & & \\
Total & 248167 & 312750 & 394790 & 524062 \\
$>65$ & 242383 & 307336 & 387365 & 516283 \\
$>85$ & 125924 & 188682 & 226738 & 342736 \\
\hline
\end{tabular}

Tableau IV

ESTIMATION DES EFFECTIFS DE POPULATION EN FRANCE DE 1990 A 2020 INSEE (1992): HYPOTHĖSES: MORTALITÉ TENDANCIELLE ; FÉCONDITE : 1,8 ; MIGRATION : + 50000 [13]

\begin{tabular}{|c|c|c|c|c|}
\hline Age & $\mathbf{1 9 9 0}$ & $\mathbf{2 0 0 0}$ & $\mathbf{2 0 1 0}$ & $\mathbf{2 0 2 0}$ \\
$60-64$ & 2892072 & 2707099 & 3712373 & 3889484 \\
$64-69$ & 2709363 & 2744468 & 2496888 & 3735896 \\
$70-74$ & 1323733 & 2474962 & 2386301 & 3355896 \\
$75-79$ & 1753738 & 2117688 & 2248765 & 2129472 \\
$80-84$ & 1210197 & 870861 & 1743380 & 1780625 \\
$85-89$ & 632561 & 827444 & 1107155 & 1283137 \\
$90-94$ & 200434 & 327247 & 274043 & 614760 \\
95 et & 41488 & 81807 & 132664 & 200688 \\
\hline
\end{tabular}

$78,6 \%$. Parmi les 13 études incluses, celles utilisant les critères du DSM III s'ajustent le mieux au modèle. Quand les seules études DSM III sont prises en compte, on obtient la formule alternative :

$\log (p)=-7,323(0,6476)+0,1150$ $(0,008197)$ âge (modèle 2$)$

$\left(\mathrm{R}^{2}=93,1 \%\right)$. Les auteurs suggèrent qu'il s'agit du meilleur modèle de relation de la démence à l'âge. Comme toutes les études DSM III ont inclus des sujets de plus de 85 ans, on dispose d'un nombre raisonnablement grand de personnes très âgées. Cela permet d'estimer avec plus de précision la prévalence de la démence aux âges les plus élevés que ne le permet chaque étude prise isolément. Le modèle montre un accroissement exponentiel, la prévalence de la démence doublant tous les six ans (figure 1). Les taux de prévalence, fournis par le modèle, pour chaque âge médian des groupes d'âges quinquennaux sont indiqués au Tableau I.

Il est possible de déduire un modèle séparé pour la maladie d'Alzheimer prise isolément à partir de quatre études qui donnent des estimations par âge et par type de démence :

$\log (p)=-16,62(1,436)+0,1646$ $(0,0176)$ âge (modèle 3$)$

avec un $\mathrm{R}^{2}$ de $82,6 \%$ et un taux de prévalence doublant toutes les 4,2 années pour plafonner après 95 ans. Les taux de prévalence par âge, fournis par le modèle, pour la maladie d'Alzheimer seule sont également indiqués dans le Tableau I. Les résultats suggèrent qu'à partir de 95 ans la maladie d'Alzheimer explique tous les cas de démence sénile. Cette anomalie est due au fait que les deux modèles sont basés sur des études différentes et que les intervalles de confiance aux âges élevés sont très larges.

\section{Estimation de la population et projection}

Les statistiques de population pour la France en 1990 proviennent du recensement général de la population de 1990 réalisé par l'INSEE et, pour les années ultérieures, des pro- 


\section{RÉFÉRENCES}

17. INSEE - Institut national de la statistique et des études économiques (1992) Recensement de la population de 1990 France métropolitaine et régions. Résultats du sondage au vingtième. INSEE résultats n 179-180-1, Démographie-Société n 13-14-15.

18. Dubuisson F, Kerjosse R. Établissements pour personnes âgées: $40 \%$ des résidents souffrent de détérioration intellectuelle. Informations rapides SESI 1993, $n^{\circ} 41$.

19. Frossard M. Le coût pour la nation. In : Fondation Nationale de Gérontologie. Démences du sujet âgé et environment. Paris : Maloine, 1985: 157-64.

20. Poulshock SW, Deimling GT. Families caring for elders in residence : issues in the measurement of burden. J Geront 1983 ; 39 : 230-9.

21. Robinson B. Validation of a care-giver strain index. J Geront 1983; 38: 344-8.

22. Zarit SH, Reever KE, Bach-Peterson J. Relatives of the impaired elderly: correlates of feelings of burden. The Gerontologist $1980 ; 20$ : 649-55.

23. Sainsbury P, Grad JA. The psychiatrist and the geriatric patient: the effects of community care on the family of the geriatric patient. J Ger Psy 1970; 4: 23-41.

24. Lezak MD. Living with the characteriologically altered brain injured patient. I Clin Psy 1978; 39 : 592-8.

25. Kiecolt-Glaser JK, Glaser R, Shuttleworth EC, Dyer CS, Ogrocki P, Speicher CE. Chronic stress and immunity in family caregivers of Alzheimer's disease victims. Psychosomatic Medicine 1987; 49 : 523-35.

26. Ritchie $\mathrm{K}$, Nargeot $\mathrm{C}$, Herguetta $\mathrm{T}$. L'hétérogénéité des stades précoces de la démence sénile de type Alzheimer à travers les observations fournies par les proches. Rev Geriatrie 1992; 17 : 251-8

27. Lucas-Blaustein MJ, Filipp CL, Dungan C, Tune L. Driving in patients with dementia. J Am Ger Soc 1988; 36 : 1087-91.

28. Ritchie K, Kildea D. Alzheimer's disease - an epidemiological standpoint. Conference proceedings Euromédecine 1991, Montpellier : 213-4.

29. Fries JF. Aging, natural death and the compression of morbidity. $N \mathrm{Engl} \mathrm{J} \mathrm{Med}$ 1980 ; 303: 130-5.

30. Fries JF. Compression of morbidity : near or far? The Milbank Quarterly 1989 ; $67: 208-32$.

31. Olshansky SJ, Carnes BA, Cassel C. In search of methuselah: estimating the upper limits to human longevity. Science 1990 ; 250: 634-40. jections INSEE pour la période 1990-2020 basées sur ce même recensement [13].

La prévalence de la démence, issue du modèle (2) puis du modèle (3) a été appliquée à la population âgée de 60 ans et plus en 1990 puis à celles prévues pour les années 2000, 2010 et 2020. Le nombre de cas de démence ainsi estimé est indiqué au Tableau II et celui de la maladie d'Alzheimer au Tableau III.

On voit, au Tableau II, qu'une augmentation nette du nombre de cas de démence sénile, modérée ou sévère, dans la population française est attendue, avec un accroissement de $21 \%$ entre 1990 et 2000, $24 \%$ entre 2000 et 2010 , et $26 \%$ entre 2010 et 2020. Entre 1990 et 2020 l'augmentation totale attendue du nombre de cas de démence chez les 60 ans et plus est de $89 \%$ alors que la population du même âge n'augmente que de $58 \%$ au cours de la même période.

Pour la démence sénile de type $\mathrm{Alz}$ heimer, qui représente approximativement $60 \%$ des cas de démences séniles, le nombre de cas prévalents augmente aussi fortement; celui-ci, estimé à 248167 en 1990, augmentant de $26 \%$ au cours de la décade suivante pour atteindre 312750 en l'an 2000, suivi d'un accroissement supplémentaire de $26 \%$ entre 2000 et 2010 , et de $33 \%$ entre 2010 et 2020 pour atteindre un nombre total de cas estimé à 524062 en 2020. A partir de la figure 2, on peut voir que la démence sénile toutes origines confondues, et la démence sénile de type Alzheimer, augmentent toutes deux fortement dans le temps.

On voit au Tableau IV une chute soudaine en 1990 des effectifs pour les personnes du groupe d'âge 70-74 ans, effectifs qui remontent pour le groupe d'âges suivant. Cette chute s'explique par l'arrivée dans ce groupe d'âge des classes creuses nées pendant la Première Guerre mondiale. Elle entraîne, par voie de conséquence, une diminution du nombre de cas de déments dans ce groupe. Dix ans plus tard, en l'an 2000, cette diminution est bien entendu transférée au groupe d'âges 80-84 ans. On note que le plus grand effectif est alors contenu dans

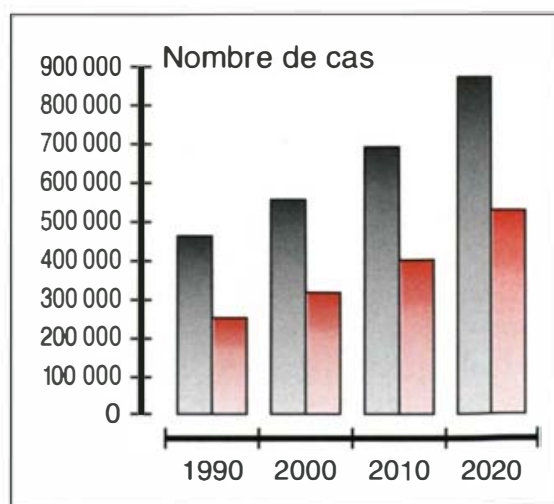

Figure 2. Projection du nombre de cas de démence en France, de 1990 à 2020. Hypothèses : mortalité tendancielle; fécondité: 1,8; solde migratoire : + 50000 par an. L'ensemble des démences est représenté par les colonnes grises, les cas de maladie d'Alzheimer par des colonnes rouges.

le groupe d'âges $65-69$ ans et non dans le groupe 60-64 ans

La figure 3 montre la distribution par groupe d'âges des cas de démences séniles pour la même année. On peut voir, alors, que les effectifs de population (Tableau IV) croisés avec les taux de prévalence (figure 1) concentrent le plus grand nombre de cas de démence dans le groupe d'âges 85-89 ans (figure 3). Dix ans plus tôt, en 1990, le plus grand nombre de cas concernait le groupe d'âges 80-85 ans.

L'application de la série de taux de prévalence de la démence sénile issue d'un modèle dérivé d'une méta-analyse des études utilisant l'algorithme diagnostique du DSM III comme procédure de dépistage des cas (modèle 2) - aux projections les plus récentes de la population, montre qu'il y avait en France en 1990 approximativement 460043 cas de démence sénile dans la population de 60 ans et plus. Pour l'an 2000, un accroissement de $21 \%$ du nombre de cas est attendu, aboutissant à un nombre de 555558 cas. Cela est bien supérieur à l'accroissement de $5 \%$ à $17 \%$ prévu par Ineichen [1] pour le Royaume-Uni de 1987 à l'an 2000, mais bien inférieur à celui de $71 \%$ prévu par Jorm et Korten [14] pour l'Australie de 1984 à l'an 2000.

L'application d'un second modèle 


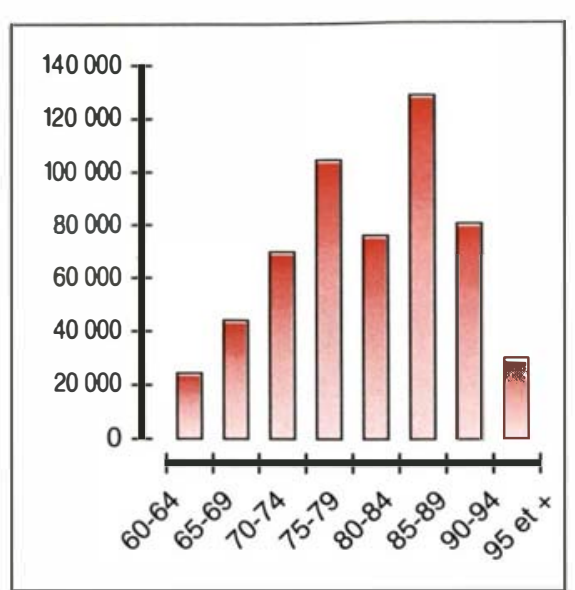

Figure 3. Distribution des cas de démence par groupes d'âges en France en I'an 2000.

— dérivé des études individualisant les différents types de démences (modèle 3) - suggère qu'en 1990 il y avait 248167 cas de démence sénile de type Alzheimer. Cette dernière estimation est nettement plus basse que celle de 300000 produite par Mas et al. [3] pour la population française de 1986. L'estimation de Mas est basée sur les projections de population de l'INSEE de 1985 et sur l'hypothèse d'un taux de prévalence de la démence sénile de type Alzheimer de $2,5 \%$ à 60 ans et plus, basée sur six études dont la moitié avait été conduite avant les années 80 et dont seulement une employait les critères DSM III. Dans ces études, peu de personnes avaient plus de 80 ans; ce qui peut conduire à un grand degré d'imprécision, à la fois pour la prévalence de la démence, et pour les niveaux de mortalité au-delà de 80 ans. Selon les prévisions de notre étude, le nombre de 300000 ne sera pas atteint avant l'an 2000. Les estimations fournies par Mas et al. semblent surestimer le nombre de cas. Comme les projections de population précédentes tendaient à sousestimer les effectifs âgés, l'effet dû aux différences dans les taux de prévalence de la démence est en partie masqué dans les écarts constatés entre les estimations présentées ici et celles de Mas et al.
On estime actuellement qu'un accroissement de $58 \%$ de la population française de 60 ans et plus d'ici l'an 2020, entraînera un accroissement de $89 \%$ du nombre de cas prévalants de démence au cours de la même période. Déjà en 1977, Gruenberg [15] attirait l'attention sur ce fait paradoxal. Les progrès médicaux, en allongeant la durée de la vie, conduisent à accroître la proportion des personnes souffrant de maladies chroniques incapacitantes au sein de la population générale. Dès 1980, Kramer [16] avait prédit la survenue d'une pandémie des troubles mentaux et des incapacités associées. Les estimations de notre étude tendent à conforter cette hypothèse.

Il faut, dès à présent, prendre en compte ce développement épidémique insidieux dans l'établissement des programmes de santé publique. Ainsi, si en 1990 le plus grand nombre de cas était concentré dans le groupe d'âge $80-85$ ans, on prévoit qu'en l'an 2000 il le sera dans le groupe d'âges 85-89 ans, entraînant une co-morbidité différente et, vraisemblablement, plus lourde. Aujourd'hui, environ $4 \%$ des personnes de plus de 60 ans vivent en institution, parmi lesquelles on estime à $40 \%$ la part de celles qui souffrent d'une démence sénile. Parmi les patients des longs séjours hospitaliers (moins de $1 \%$ des personnes de plus de 60 ans), la proportion atteindrait $75 \%[17,18]$. A partir de ces chiffres, on peut estimer que $30 \%$ à $40 \%$ environ des personnes atteintes de démence sénile sont placées en institution.

En 1984, on estimait déjà le coût de la dépendance liée à la démence à 7 milliards de francs [19] et encore le problème ne se limite pas aux seuls soins des personnes âgées démentes. De nombreuses études épidémiologiques ont en effet montré que les membres de l'entourage prenant en charge les personnes démentes, les care-givers, présentent eux-mêmes un risque de maladies physiques et mentales significativement plus élevé, largement attribuable au stress accompagnant le rôle de soignant [20-25]. En outre, plusieurs études récentes en France [26] et aux États-Unis [27] suggè- rent que les sujets atteints de démence sénile sont responsables, dans des proportions anormales, d'accidents de la circulation.

La précision des estimations données ici doit être doublement examinée, à savoir celle de l'estimation de la prévalence et celle des projections de population.

Les estimations de la prévalence retenues pour l'ensemble de la période 1990-2020 ont été faites à partir d'études épidémiologiques relativement récentes. Il reste évidemment un certain degré d'erreur, principalement dû à l'existence de "faux positifs", en terme de diagnostic, et au fait que les effectifs sont d'autant plus petits que les groupes d'âges considérés sont élevés. On doit se rappeler que les estimations sont ici fournies uniquement pour les cas de démence modérée et sévère.

Le scénario retenu présume que les prévalences par sexe et par âge de la démence sénile resta inchangée au cours de la période étudiée, bien que des effets de cohorte puissent exister, en particulier s'il se confirme qu'un fort niveau de scolarité diminue le risque de démence sénile.

Les taux de prévalence de la démence sont sûrement assez fiables jusqu'à 90 ans et leur ajustement par un modèle exponentiel satisfaisant. Au-delà, il y a une grande incertitude concernant la relation de la prévalence avec l'âge et il est possible que la croissance de la prévalence avec l'âge ralentisse. Un modèle logistique est peut-être mieux adapté, mais les observations sont trop rares pour qu'on puisse le vérifier [28]. Si aujourd'hui ce point peut être négligé, compte tenu du faible effectif de la population très âgée, la croissance actuelle du nombre de centenaires va le rendre crucial dans les décennies à venir.

La précision des estimations de la population âgée en l'an 2020 n'est sûrement pas bien grande. Il a toujours été difficile pour les démographes d'imaginer une poursuite de la chute de la mortalité comme le montre l'étude des différentes projections INSEE (1975-2020, 1985-2040, 1990-2020) et l'ampleur des révisions. Le chiffre magique de 
85 ans popularisé par Fries [29, 30] comme limite théorique et par Olshansky et al. [31] comme limite pratique à la croissance de l'espérance de vie ont sûrement une part non négligeable dans la prudence traditionnelle des démographes, malgré des évidences empiriques sur la prévisibilité de nouveaux gains sur la mortalité.

Cette prudence a pu conduire à une grave sous-estimation du nombre futur de personnes âgées, particulièrement des très âgés. En effet, la progression attendue des effectifs est d'autant plus marquée que l'on monte plus haut dans l'échelle des âges. Ce point est essentiel, car sur les 7 à 8 millions d'habitants supplémentaires que comptera la France en 2020, six millions viendront gonfler la population de 60 ans ou plus [13]. L'incertitude se déplace du nombre des naissances au nombre des très âgés. On note, par exemple, qu'entre 1975 et 1990 la prévision de la population âgée de 85 ans et plus en l'an 2000 a varié de 865000 à 1236000 , soit une correction de $43 \%$.

On peut se demander si on n'est pas passé d'un extrême à l'autre. En 1975, on prévoyait moins d'une année de gain d'espérance de vie à la naissance en 30 ans, et maintenant, en 1990, on en prévoit plus de 5 . En 1975, l'hypothèse tendancielle prolongeait essentiellement le ralentissement de la croissance de l'espérance de vie observé dans les années 1960. En 1990, l'hypothèse tendancielle prolonge la très forte croissance observée au cours des deux dernières décennies. Faute d'informations à caractère épidémiologique, il est très difficile pour les démographes de s'écarter des tendances passées, même si l'hypothèse tendancielle est sûrement, par définition, celle qui a le moins de chance de se réaliser.

Dans ces conditions, et compte tenu du fait que les projections de population reposent essentiellement sur une hypothèse tendancielle d'évolution de la mortalité, il est important de se limiter dans les projections à des horizons peu éloignés, d'une dizaine d'années, et de repérer précocement tout changement de tendance dans l'évolution de la morta- lité, en particulier aux âges les plus élevés.

\section{Conclusions}

La précision des estimations du nombre de cas de démence sénile présentées ici est inconnue. Toutefois, l'application d'un modèle de la prévalence de la démence sénile issu d'études récentes ayant utilisé des procédures de diagnostic internationalement reconnues, et portant sur des effectifs satisfaisants dans les groupes d'âges les plus élevés — aux données du recensement général de la population de 1990 fournit vraisemblablement une excellente estimation du nombre actuel (en 1990) de cas de démence dans la population de 60 ans ou plus. Il y aurait 460000 cas de démence dans la population de 60 ans et plus en 1990, dont 248000 cas de démence sénile de type Alzheimer. Au-delà, l'application aux projections de population les plus récentes réalisées par l'INSEE fournit probablement les meilleures estimations que l'on puisse obtenir aujourd'hui. Elles sont sûrement assez fiables pour l'an 2000, mais au-delà, en 2010 ou en 2020, l'incertitude grandit. Ainsi, il y aurait de 533000 à 556000 cas de démence en France dans la population de 60 ans et plus en l'an 2000 , selon l'hypothèse basse de 1985 ou l'hypothèse tendancielle de 1990, dont respectivement de 292000 à 313000 cas de démence sénile de type Alzheimer. Bien que les estimations obtenues pour l'an 2000 soient plus faibles que celles précédemment calculées, l'accroissement attendu du nombre de personnes âgées souffrant de démence sénile devra être pris en compte dans l'allocation des ressources si on veut faire face à cette nouvelle épidémie

\section{TIRÉS A PART}

\section{K. Ritchie}

\section{Summary}

Senile dementia in France: estimating the current burden and projecting future trends

An estimate of the number of persons in France presently suffering from senile dementia has been made using a meta-analysis derived from recent epidemiological studies in conjunction with population statistics from the 1990 INSEE census. The results suggest that the number is less than has previously been estimated, but that significant increases can be expected in coming years if no efficient treatment is found for these disorders and the life expectancy of persons with dementia is not reduced. The importance of these observations in terms of public health planning extends beyond the help required for the elderly dementing person, to the implications of such an epidemic for family care-givers. 